\title{
Short communication: Patterns of dairy consumption in free-living children and adolescents
}

\author{
Benjamin P. Green, ${ }^{1}$ Louise Turner, Emma Stevenson, and Penny L. S. Rumbold \\ Department of Sport, Exercise and Rehabilitation, Faculty of Health and Life Sciences, Northumbria University, Northumberland Building, \\ Newcastle upon Tyne NE1 8ST, United Kingdom
}

\section{ABSTRACT}

According to national survey data, dairy food consumption has fallen in recent years and declines further with age, especially from childhood to adolescence. Dietary surveys typically rely on retrospective dietary assessment methods and use broad age groupings (4-10 yr; 11-18 yr), making it challenging to differentiate between middle-childhood and adolescence. Consequently, there is a need to assess dairy food consumption during middle-childhood and adolescence using more robust dietary assessment tools. Therefore, the present study aimed to describe and compare patterns of dairy consumption throughout middle-childhood and adolescence. Dairy food consumption was assessed during school term-time over 4 consecutive days, including 2 weekdays and 2 weekend days, in a sample of free-living children $(9-11 \mathrm{yr}, \mathrm{n}=40)$ and adolescents $(15-18 \mathrm{yr}, \mathrm{n}=35)$. For children, free-living dairy intake was evaluated through parental-weighed food records, and for adolescents, a combined weighed self-reported food record and 24-h dietary recall technique was utilized. Food records were explored to determine types, amounts, and frequency of dairy food consumption, and were analyzed for differences between middle-childhood and adolescence using a between group $2 \times 2($ age $\times$ sex) ANOVA. Descriptive data suggested that milk was the most popular dairy product consumed by both children and adolescents. Statistical analysis revealed a main effect for sex on total milk consumption $(\mathrm{mL})$ and number of daily milk portions consumed. No interaction or main effect was present for any other variable. The present study indicates that independent of age, boys consumed greater amounts of milk compared with girls. Contrary to existing literature, findings suggest no difference in milk-based dairy consumption between middle-childhood and adolescence.

Key words: dairy, milk, adolescents, children

Received November 26, 2014.

Accepted February 8, 2015.

${ }^{1}$ Corresponding author: benjamin.green@northumbria.ac.uk

\section{Short Communication}

Milk-based dairy foods represent a nutrient-dense foodstuff. Housing an extensive assortment of nutrients, milk-based dairy foods contribute significantly to intakes of high-quality proteins, micronutrients, and numerous bioactive constituents. Consequently, milkbased dairy foods exert the potential to affect human health (Fiorito et al., 2006), and their inclusion as a component of a healthy balanced diet is widely recognized.

Despite the acknowledged benefits of dairy foods, per capita consumption has declined in recent years and declines further with increasing age, particularly throughout adolescence (Bates et al., 2014). Nonetheless, much of our knowledge concerning dairy consumption is centered on national surveillance data. Originally established in 1992, the National Diet and Nutrition Survey remains the only surveillance program in the United Kingdom that provides a nationally representative assessment of dietary habits of the general population (1.5-3 yr, $4-10$ yr, $11-18$ yr, $16-64$ yr, and $\geq 65$ yr). Dietary surveys typically rely on retrospective assessment methods, which pose complications of misreporting error and are not therefore entirely robust. Furthermore, the wide-ranging age groupings (e.g., 4-10 yr, 11-18 yr) make it difficult to differentiate between consumption in middle-childhood and adolescence. It is therefore important to assess dairy consumption throughout this period, exercising more finite age boundaries and more robust dietary assessment tools. Accordingly, the present study aimed to examine and compare patterns of free-living dairy food consumption among a sample of children (9-11 yr) and adolescents (15-18 yr).

This study comprised a convenience sample of participants aged 9 to $11 \mathrm{yr}$ ( 15 boys and 25 girls) and 15 to 18 yr (20 males and 20 females), recruited from a local primary and secondary school, respectively, in northeast England. The study was conducted according to the guidelines laid down in the 2013 Declaration of Helsinki (WMA, 2013), and all procedures involving human subjects were approved by the Faculty of Health and Life Sciences Ethics Committee at the University 
of Northumbria (Newcastle upon Tyne, UK). All participants provided parental written informed consent before data collection.

Free-living dietary intake was evaluated during school term-time over 4 consecutive days, including 2 weekdays and 2 weekend days. Adolescent (15-18 yr) free-living dietary intake was evaluated by using a combined weighed self-reported food record and 24-h dietary recall technique, used previously with adolescent populations (Rumbold et al., 2011). The reporting accuracy of this approach has been demonstrated to be an effective method for dietary assessment in adolescents (confidence intervals for bias ranging from 0.00 to 0.95 megajoules; Rumbold et al., 2011). For children (9-11 yr), free-living dietary intake was evaluated through parental-weighed food records. During periods of dietary data collection, a parent or guardian was requested to report all food and drink items consumed, with assistance from the participating child where necessary. Parental food records have successfully been used when reporting dietary habits of children (4-10 yr) and are comparable to measures of energy expenditure using doubly labeled water (Bates et al., 2014).

During periods of dietary data collection, adolescent participants and parents or guardians of the participating child were requested to give comprehensive recordings of all food and drink items consumed, weighing items before and after consumption (if leftovers were present). Additional information deemed necessary included methods of preparation and cooking, names of branded products, and condiment use. For homemade dishes, participants were asked to record individual ingredients and quantities for the whole dish, along with a brief description of cooking method and how much of the dish they consumed. Reported dietary intakes were subsequently explored to determine types, amounts, and frequency of dairy food consumption.

Trained research staff examined all food records, which were subsequently analyzed using the nutritional software package Nutritics (Nutritics Professional v3.09, Nutritics, Dublin, Ireland). Food records were explored to determine types, amounts, and frequency of dairy food consumption. Dairy food categories in the present study included milk (whole, reduced-fat, fat-free, and flavored milk), yogurt (all yogurt types), cheese (all cheese types), butter, ice cream, cream, and custard. For the present study, a serving of dairy equated to $150 \mathrm{~mL}$ of milk, $25 \mathrm{~g}$ of cheese, $120 \mathrm{~g}$ of yogurt, $5 \mathrm{~g}$ of butter, $75 \mathrm{~g}$ of ice cream, $15 \mathrm{~mL}$ of cream, and $75 \mathrm{~g}$ of custard. Patterns of dairy consumption were dichotomized according to overall consumption and average daily servings.

The statistical software package SPSS (version 21.0; SPSS Inc., Chicago, IL) was used for all data analysis.
Descriptive data are presented as means and standard deviations (SD). Results are presented by groups according to sex and age (boys 9-11 yr; boys 15-18 yr; girls 9-11 yr; girls 15-18 yr). Differences in overall consumption and average daily servings of milk, cheese, yogurt, butter, ice cream, cream, and custard were analyzed using a between group $2 \times 2$ (age $\times$ sex) ANOVA. Following a significant interaction, the least significant difference (LSD) post hoc test was used to determine the location of variance, and all $P$-values reported were adjusted for multiple comparisons. Statistical significance was accepted at $P<0.05$ for all analysis.

In total, data are presented for 75 participants [9-11 yr ( $\mathrm{n}=15$ boys and 25 girls) and $15-18$ yr ( $\mathrm{n}=18$ males and 17 females)]. Three participants were excluded because of unsatisfactory food record completion, and 2 withdrew their interest in participating during data collection. Age, stature, body mass, and body mass index for participating children were 9.4 (SD 0.5) $\mathrm{yr}, 1.41(\mathrm{SD} 0.07) \mathrm{m}, 33.49$ (SD 7.46) $\mathrm{kg}$, and 16.8 (SD $3.1) \mathrm{kg} / \mathrm{m}^{2}$, respectively, and 16.1 (SD 1.0) yr, 1.71 (SD $0.1) \mathrm{m}, 66.2(\mathrm{SD} 15.1) \mathrm{kg}$, and $22.6(\mathrm{SD} 3.5) \mathrm{kg} / \mathrm{m}^{2}$ for adolescents, respectively. The body mass index for participating children and adolescents indicates that participants were classified as normal weight according to the UK 1990 age and sex reference population (Cole et al., 1995). Data concerning types, amounts, and frequencies of consumed dairy foods are presented in Table 1 by age and sex, dichotomized according to overall consumption and average daily servings.

Statistical analysis revealed a main effect for sex on overall milk consumption $\left(\mathrm{F}_{1,71}=7.07, P=0.010\right)$ and daily milk portions $\left(\mathrm{F}_{1,71}=6.79, P=0.011\right)$, indicating that independent of age, boys consumed greater amounts of milk compared with girls (Table 1). No interaction or main effect for any other variable (cheese, yogurt, butter, ice cream, cream, or custard) was evident. Total daily dairy servings did not differ significantly between ages $\left(\mathrm{F}_{1,71}=2.12, P=0.150\right)$ or sexes $\left(\mathrm{F}_{1,71}=2.48, P\right.$ $=0.120)$, and conformed with current dietary recommendations (Dror and Allen, 2014). Patterns of milk and dairy food consumption remained widely stable among girls and female adolescents (Table 1). For boys and adolescent males, we noted a downward trend of milk and dairy food consumption with increasing age, although differences were not significant (Table 1). The major contributing source of dairy was milk, consumed by $91 \%$ of participants (across all ages and sexes), followed by cheese and yogurt consumption, at 60 and $56 \%$, respectively.

The main observations of this study were that, independent of age, boys consumed greater amounts of milk compared with their female counterparts. This was apparent for both overall milk consumption and daily 


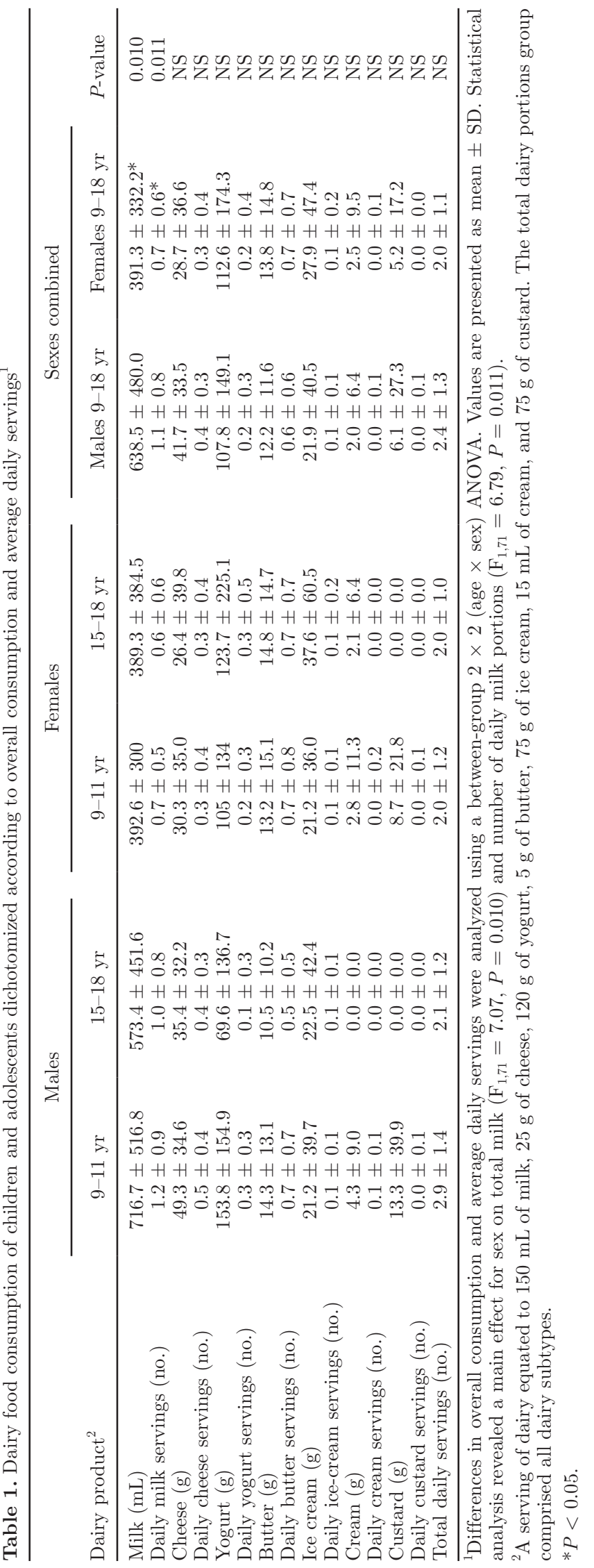

milk servings. These findings are consistent with other cross-sectional data showing that boys consume greater quantities of milk than girls (Forshee and Storey, 2003; Novotny et al., 2003; Mensink et al., 2007). Contrary to existing literature (Morton and Guthrie, 1998; Cavadini et al., 2000; Baird et al., 2012; Parker et al., 2012; Bates et al., 2014), however, the results reported here suggest that there is no difference in dairy consumption (types, amounts, and frequency) between middle-childhood and adolescence. In the present study, daily dairy servings complied with current dietary recommendations (2-3 servings daily; Dror and Allen, 2014) for all age groupings and between sexes. However, consumption patterns among boys (15-18 yr) and girls (9-11 and 15-18 yr) were on the lower boundary of the recommended daily servings. These findings are consistent with previous studies that have shown poor compliance of dairy consumption among US children and adolescents (Cavadini et al., 2000; Fiorito, et al., 2006). This is of particular concern because dairy food avoidance and low dairy consumption could be disadvantageous, particularly among adolescents, leading to nutritional and health-related implications (Nicklas, 2003). Milkbased dairy foods represent a nutrient-dense foodstuff and encompass a host of bioactive constituents pertinent to human health (Fiorito et al., 2006). Daily consumption of dairy contributes significantly to intakes of highquality proteins and various essential micronutrients, such as calcium, riboflavin, and vitamin D (if fortified), helping children and adolescents achieve nutrient intake recommendations and augment dietary quality (Weaver et al., 2013).

Although the findings reported here cannot be used to elucidate motivations for differences in dairy food consumption between boys and girls, numerous observations indicate that participants associate the consumption of dairy with increased BW (Wham and Worsley, 2001; Nolan-Clark et al., 2011). Research suggests that female adolescents decrease dairy food consumption due to concerns regarding BW gain and the notion that dairy foods are fattening (Neumark-Sztainer et al., 1997; Gulliver and Horwath, 2001). Indeed, dairy foods contain saturated fats, and historical views have linked elevated plasma cholesterol following dairy consumption to increased cardiovascular risk (Soerensen et al., 2014; Tunick and Van Hekken, 2014). The belief that dairy foods are fattening may have contributed to the assumption that dairy is a factor in obesity (Elwood et al., 2010) and consumption should consequently be limited. However, trends concerning the incidence of overweight and obesity are concurrent with declining levels of dairy consumption, suggesting that dairy may confer a direct or indirect protective effect against adiposity. Following an expanding body of research, a 
potential role of milk and dairy consumption in the prevention of BW gain has emerged (Barba et al., 2005; Moore et al., 2008; Abreu et al., 2014). Research concerning the effect of dairy foods on mechanisms facilitating the relationship between dairy and adiposity, however, is sparse, particularly within the free-living environment. Further research is therefore warranted to verify the magnitude of the effect, if any, of dairy food consumption among children and adolescents on the aforementioned mechanisms.

In agreement with figures from Australian children and adolescents (2-16 yr; Baird et al., 2012), the National Health and Nutrition Examination Survey (Moore et al., 2008), and the National Diet and Nutrition Survey (Bates et al., 2014), milk was the most favored dairy food among children and adolescents in the current study, consumed by $91 \%$ of participants. This was followed by cheese and yogurt, consumed by 60 and $56 \%$ of participants, respectively. In general, overall consumption and daily servings of milk and cheese were more pronounced among boys and adolescent males compared with their female counterparts; however, the opposite was apparent for yogurt and ice cream. Nonetheless, daily servings remained similar between sexes and ages (Table1).

Caution should be taken when extrapolating the results of this study because the findings are limited to a relatively small population of children and adolescents in northeast England. Further, limitations of this study warrant consideration. Data regarding free-living dairy consumption were obtained through self-reported records. A major drawback of participant-centered data collection is that such approaches present opportunities for bias and misreporting (Illner et al., 2010). In the present study, 2 methodological approaches were used to explore dairy consumption that we believe to be a novel aspect of the study. Adolescent free-living dairy intake was evaluated utilizing a combined weighed selfreported food record and 24-h dietary recall technique, and through parental centered food records for children, in an attempt to combat opportunities for bias and misreporting. The reporting accuracy of this combined technique indicated a slight bias (4.2\%) toward over-reporting, albeit negligible and in accordance with previous literature, thus demonstrating this approach to be an effective method to collect dietary information in adolescents. Furthermore, parental centered food records have successfully been used when reporting dietary habits of children (4-10 yr) and have shown to be comparable to records based on doubly labeled water (Bates et al., 2014).

In summary, these findings contribute valuable information for researchers and practitioners regarding dairy food consumption between middle-childhood and adolescence in the United Kingdom. Findings reported here indicate that milk was the most favored dairy food among children and adolescents. Furthermore, independent of age, boys consumed greater amounts of milk compared with girls. No difference between middlechildhood and adolescence was observed, affirming that dairy food consumption remains stable throughout this period. We confirm that wide age groupings make it challenging to differentiate between consumption throughout middle-childhood and adolescence and encourage future dietary surveillance surveys to exercise more finite age boundaries in an attempt to better represent the dietary habits of differing populations.

\section{ACKNOWLEDGMENTS}

The authors thank all volunteers for participating in the study. We also thank the Dairy Council UK (London) for the external funding for the study.

\section{REFERENCES}

Abreu, S., R. Santos, C. Moreira, P. C. Santos, S. Vale, L. SoaresMiranda, R. Autran, J. Mota, and P. Moreira. 2014. Relationship of milk intake and physical activity to abdominal obesity among adolescents. Pediatr. Obes. 9:71-80. http://dx.doi.org/10.1111/ j.2047-6310.2012.00130.x.

Baird, D. L., J. Syrette, G. A. Hendrie, M. D. Riley, J. Bowen, and M. Noakes. 2012. Dairy food intake of Australian children and adolescents 2-16 years of age: 2007 Australian National Children's Nutrition and Physical Activity Survey. Public Health Nutr. 15:2060-2073. http://dx.doi.org/10.1017/S1368980012001176.

Barba, G., E. Troiano, P. Russo, A. Venezia, and A. Siani. 2005. Inverse association between body mass and frequency of milk consumption in children. Br. J. Nutr. 93:15-19.

Bates, B., A. Lennox, A. Prentice, C. Bates, P. Page, S. Nicholson, and G. Swan. 2014. National Diet and Nutrition Survey: Results from Years 1, 2, 3 and 4 (combined) of the Rolling Programme 2008/09 - 2011/12. Public Health England, London, UK. Accessed May 15, 2014. https://www.gov.uk/government/statistics/national-diet-and-nutrition-survey-results-from-years-1-to-4-combined-of-therolling-programme-for-2008-and-2009-to-2011-and-2012.

Cavadini, C., A. M. Siega-Riz, and B. M. Popkin. 2000. US adolescent food intake trends from 1965 to 1996. Arch. Dis. Child. 83:18-24. http://dx.doi.org/10.1136/adc.83.1.18.

Cole, T. J., J. V. Freeman, and M. A. Preece. 1995. Body mass index reference curves for the UK, 1990. Arch. Dis. Child. 73:25-29. http://dx.doi.org/10.1136/adc.73.1.25.

Dror, D. K., and L. H. Allen. 2014. Dairy product intake in children and adolescents in developed countries: Trends, nutritional contribution, and a review of association with health outcomes. Nutr. Rev. 72:68-81. http://dx.doi.org/10.1111/nure.12078.

Elwood, P. C., J. E. Pickering, I. D. Givens, and J. E. Gallacher. 2010. The consumption of milk and dairy foods and the incidence of vascular disease and diabetes: An overview of the evidence. Lipids 45:925-939. http://dx.doi.org/10.1007/s11745-010-3412-5.

Fiorito, L. M., D. C. Mitchell, H. Smiciklas-Wright, and L. L. Birch. 2006. Dairy and dairy-related nutrient intake during middle childhood. J. Am. Diet. Assoc. 106:534-542. http://dx.doi. org/10.1016/j.jada.2006.01.005.

Forshee, R. A., and M. L. Storey. 2003. Total beverage consumption and beverage choices among children and adolescents. Int. J. Food Sci. Nutr. 54:297-307. 
Gulliver, P., and C. Horwath. 2001. Women's readiness to follow milk product consumption recommendations: Design and evaluation of a 'stage of change' algorithm. J. Hum. Nutr. Diet. 14:277-286. http://dx.doi.org/10.1046/j.1365-277x.2001.00295.x.

Illner, A. K., U. Nöthlings, K. Wagner, H. Ward, and H. Boeing. 2010. The assessment of individual usual food intake in large-scale prospective studies. Ann. Nutr. Metab. 56:99-105.

Mensink, G. B., C. Kleiser, and A. Richter. 2007. Food consumption of children and adolescents in Germany. Results of the German Health Interview and Examination Survey for Children and Adolescents (KiGGS). Bundesgesundheitsblatt Gesundheitsforschung Gesundheitsschutz 50:609-623. http://dx.doi.org/10.1007/ s00103-007-0222-x.

Moore, L. L., M. R. Singer, M. M. Qureshi, and M. L. Bradlee. 2008. Dairy intake and anthropometric measures of body fat among children and adolescents in NHANES. J. Am. Coll. Nutr. 27:702-710.

Morton, J. F., and J. F. Guthrie. 1998. Changes in children's total fat intakes and their food group sources of fat, 1989-91 versus 1994-95: Implications for diet quality. Fam. Econ. Rev. 11:44-57.

Neumark-Sztainer, D., M. Story, L. B. Dixon, M. D. Resnick, and R. W. Blum. 1997. Correlates of inadequate consumption of dairy products among adolescents. J. Nutr. Educ. 29:12-20. http:// dx.doi.org/10.1016/S0022-3182(97)70141-9.

Nicklas, T. A. 2003. Calcium intake trends and health consequences from childhood through adulthood. J. Am. Coll. Nutr. 22:340356. http://dx.doi.org/10.1080/07315724.2003.10719317.

Nolan-Clark, D. J., E. P. Neale, Y. C. Probst, K. E. Charlton, and L. C. Tapsell. 2011. Consumers' salient beliefs regarding dairy products in the functional food era: A qualitative study using concepts from the theory of planned behaviour. BMC Public Health 11:843.
Novotny, R., C. Boushey, M. A. Bock, L. Peck, G. Auld, C. M. Bruhn, D. Gustafson, K. Gabel, J. K. Jensen, S. Misner, and M. Read. 2003. Calcium intake of Asian, Hispanic and white youth. J. Am. Coll. Nutr. 22:64-70. http://dx.doi.org/10.1080/07315724.2003.1 0719277.

Parker, C. E., W. J. Vivian, W. H. Oddy, L. J. Beilin, T. A. Mori, and T. A. O'Sullivan. 2012. Changes in dairy food and nutrient intakes in Australian adolescents. Nutrients 4:1794-1811.

Rumbold, P. L. S., A. St Clair Gibson, E. Stevenson, and C. J. DoddReynolds. 2011. Agreement between two methods of dietary data collection in female adolescent netball players. Appetite 57:443447. http://dx.doi.org/10.1016/j.appet.2011.06.013.

Soerensen, K. V., T. K. Thorning, A. Astrup, M. Kristensen, and J. K. Lorenzen. 2014. Effect of dairy calcium from cheese and milk on fecal fat excretion, blood lipids, and appetite in young men. Am. J. Clin. Nutr. 99:984-991. http://dx.doi.org/10.3945/ ajcn.113.077735.

Tunick, M. H., and D. L. Van Hekken. 2014. Dairy products and health: Recent insights. J. Agric. Food Chem. http://dx.doi. org $/ 10.1021 /$ jf5042454

Weaver, C., R. Wijesinha-Bettoni, D. McMahon, L. Spence, E. Muehlhoff, and A. Bennett. 2013. Milk and dairy products in human nutrition. Page 404 in Milk and Dairy Products as Part of the Diet. Food and Agriculture Organization of the United Nations. Accessed Jul. 17, 2014. http://www.fao.org/docrep/018/i3396e/ i3396e.pdf.

Wham, C., and A. Worsley. 2001. Demographic differences in usage and attitudes to milk. NZ Diet. Assoc. J. 55:18-21.

WMA. 2013. World Medical Association Declaration of Helsinki: Ethical principles for medical research involving human subjects. JAMA 310:2191-2194. 10.1001/jama.2013.281053. 